DISCUSSION:

Paper:

Author:

WANG:

DE BOER:

RUSSELL:

DE BOER:

LEQUEUX:

DE BOER:

TOBIN:

DE BOER:

HELFLAND:

DE BOER:

Paper:

Author:

MEURER:

SAUVAGE:

\title{
THE INTERSTELLAR MEDIUM
}

The interstellar medium of the Magellanic Clouds from absorption lines Klaas S. de Boer

In the case when the ISM is clumpy, the study of the absorption lines gives information along particular lines of sight only. It would be difficult to access the real structure of the medium.

Yes, it is true. We have to compare the results from absorption line studies (Ly $\alpha$ ) with those from a $21 \mathrm{~cm}$. We are looking forward to the AT to get high resolution radio maps of the relevant lines of sight in the MC's.

Please comment on the expected errors in your $C$ and $N$ abundances in the ISM of the $L M C$.

The abundance of these elements were derived from emission lines and it is not quite fair to include them in the figure.

From your figure it appears that silicon might be less depleted in the LMC than in the SMC compared to other elements. If real this may be of high significance as silicates are one of the main compounds of dust. Is the difference significant?

Unfortunately, the Si determination is rather uncertain. You must also have noticed the Fe is missing. This is because the Fe II lines are so strong that the various absorption components are difficult to disentangle. With Si one has the weak $1808 \AA$ line as a fair indicator.

Savage claims that interstellar titanium is very smoothly distributed in both the disc and halo of the Milky Way, and indeed can be used as a 'secondary' distance indicator, e.g. for determining distances to high-latitude $B$ stars. Is there any evidence that titanium is smoothly distributed in the Clouds?

The only $\mathrm{Ti}$ data available is the upper limit to $\mathrm{R} 136$. So there is lots of observing to do!

You cited the NV lines as evidence for a corona around the $L M C$; is there any reason not to associate this with coronal gas permeating the ISM of the galaxy rather than as a corona surrounding it?

Yes, it is well possible that there is very hot gas in the LMC itself. The case for coronae around the Clouds is not as strong as that for hot gas around the Milky Way (see IAU Symp. 108).

Dust distribution and temperature in the Magellanic Clouds interstellar medium

$$
\text { M. Sauvage, L. Vigroux }
$$

It is good to see that $\mathrm{vf}(12 \mu \mathrm{m}) / f(F I R)$ correlates with log [O/H]. Can you comment on how good the Balmer decrement is as a dust indicator considering that Balmer decrement reddening parameter does not correlate with $\log [O / H]$ nor $f(F I R) f_{B}$ nor $(U-B)($ Salzer \& McAlpine 1988, Kunth \& Sevre 1986)?

This shows that the dust is a highly heterogeneous phase so that indicators based on a given wavelength do not probe the same specie. Furthermore, the effect of metallicity is

441

R. Haynes and D. Milne (eds.), The Magellanic Clouds, 441-443.

(C) 1991 IAU. Printed in the Netherlands. 
LEQUEUX: $\quad$ We (E. Le Coarer, L. and M.L. Prévot and I) have new far-UV extinction curves for LMC stars outside the 30 Dor area. The systematic difference between 30 Dor and the rest becomes less marked than in Fitzpatrick's work.

MEABURN:

KOORNNEEF

Paper:

Author:

KENNICUTT:

MEABURN:

WANG:

MEURER:

Paper:

Author:

KENNICUTT

LEQUEUX:

DE BOER: only of second order (see Sauvage et al. 1990) since neither $f(F I R) f_{B}$ nor $v f(12 \mu m) f_{B}$ correlates with $\log [\mathrm{O} / \mathrm{H}]$. The abundance of a given dust specie is not primarily ruled by the metallicity.

Far infra-red emission lines can make some contribution to all of the IRAS bands. I suppose we must await spectra before the amount of contamination can be assessed.

On the issue of variations of the interstellar extinction law from region to region in the $L M C$, it has to be noted that since the work of Fitzpatrick, IRAS has shown that there are large, random variations in the galactic foreground extinction with an additional systematic increase from the east to the west of the LMC. The result will be that reddened LMC stars will, statistically, have a larger galactic component with the consequence that the derived extinction will be biased in the direction of a Galactic type law. As a result, the concept of 30 Doradus versus non-30 Doradus extinction is almost certainly a serious oversimplification.

\section{Studies of the LMCs using optical interstellar emission lines J. Meaburn}

I have two comments concerning your estimate of 40 SNRs in 30 Dor. Chu and I find that the fast expanding shells are strongly concentrated to the core of $30 \mathrm{Dor}$, so extrapolating the observed number of shells in the core to the whole nebula may overestimate the total. Also, it is quite likely that many of the fast shells are young, wind-driven shells, in addition to SNRs.

I take your point to some extent about the total number over the area of 30 Dor. However, I cannot think of any wind-generated, pressure-driven bubbles in the Galaxy of $15 \mathrm{pc}$ diameter expanding at $260 \mathrm{~km} \mathrm{~s}^{-1}$. But, I agree in principle they could occur.

One comment. Most of the high velocity component observed in the $H \alpha$ line emission can be explained with the turbulent motion in the hot gas. Since the hot gas $\left(\sim 10^{6} \mathrm{~K}\right)$ dominates the space in the nebula, the motion of warm or cold gas, existing in sheets or clouds, is driven by the turbulent motion in the hot gas. The resultant motion may appear to be coherent at small scales.

The general morphology of the greater 30 Dor region with small bubbles in the centre and two great bubbles symmetric about the centre, is similar in morphology to MacLow and McCray's bubble blow out models.

Molecular gas in the Small Magellanic Cloud Monica Rubio

Could you or one of the other speakers comment on the propspects for directly detecting $H 2$ in absorption in the SMC, perhaps with the new UV space observatories? A factor of 20 effect should be quite unambiguous.

With the HST it is impossible because it is not sensitive below $120 \mathrm{~nm}$ whereas the $\mathrm{H}_{2}$ absorption lines lie near and below $110 \mathrm{~nm}$.

It might be possible to detect $\mathrm{CO}$ in the UV absorption lines. 
Paper:

Author:

LORTET:

PETTINI:

HELFAND:

PETTINI:
Studies of the ISM in the LMC using SN1987A Max Pettini

I would draw attention to the star which is west of SN 1987A on the first (inner) ring. It is a red supergiant and it would be very interesting to have photometry and spectra to derive its reddening, which would allow us to locate it along the line of sight relative to the SN.

I agree.

Is it possible to measure differences between the SN spectrum and the echo spectrum in order to learn something about the dust properties?

In principle, yes. In practice, the echo spectrum I showed was obtained under conditions too poor to allow such a comparison to be made. However, as the rings should remain bright for several years, it will be possible to obtain better spectra in the future. 